\title{
Could mycobacterial MelF protein (Rv1936) be used as a potential drug target?
}

\author{
Promod K. Mehta*,1, Renu Dharra ${ }^{1}$ \& Mahesh Kulharia² \\ ${ }^{1}$ Center for Biotechnology, Maharshi Dayanand University, Rohtak-124001, India \\ ${ }^{2}$ School of Basic \& Applied Science, Central University of Punjab, Bathinda-151001, India \\ *Author for correspondence: Fax: +91 1262 274640; pkmehta3@hotmail.com
}

“'the inhibitors targeting MelF (anti-ROS/RNS machinery) would open up fresh avenues pertaining to the design of adequate ROS generators (both exogenous and endogenous), which may also improve the efficacy of current ATDs."

First draft submitted: 1 June 2018; Accepted for publication: 20 June 2015; Published online: 21 September 2018

Keywords: M. marinum • M. tuberculosis • MelF

Macrophages produce antimicrobial reactive oxygen species (ROS) and reactive nitrogen species (RNS) through $\mathrm{NADPH}$ oxidase (NOX2/gp91 ${ }^{\text {phox }}$ ) and inducible nitric oxide synthase in response to mycobacterial infections [1]. In general, mycobacteria are resistant to ROS, but RNS inhibit growth and even kill mycobacteria within the activated macrophages. Mycobacterium tuberculosis is also continually exposed to endogenous ROS including the production of superoxide radicals as part of normal aerobic respiration [2]. In fact, M. tuberculosis has developed several defense mechanisms to counteract ROS stress, for example, lipid enriched cell wall, maintenance of cytosolic redox homeostasis, genes involved in repair and protection of DNA/protein as well as several ROS scavenger genes, including katG (Rv1908c), SodA (Rv3846), sodC (Rv0432), ahpC (Rv2428), ahpE (Rv2238c), melF (Rv1936), etc. $[3,4,5]$. Notably, global regulator $o x y R$ is inactive in $M$. tuberculosis, but is active in other pathogenic mycobacterial species including $M$. marinum. Pathways involved in mycobacterial resistance to RNS include the generation of RNS scavenger genes such as noxR1 (Rv2997), noxR3 (Rv1500), dlaT (Rv2215), msrA (Rv0137c) and cysH (Rv2392), DNA repair as well as protein degradation in the proteasome [3]. Moreover, peroxynitrite is produced by SOD in the presence of $\mathrm{H}_{2} \mathrm{O}_{2}$ and nitric oxide, connecting the two important mechanisms of oxidative and nitrosative stress-mediated bacterial cell death. $M$. tuberculosis possesses inherent resistance to peroxynitrite as compared with less pathogenic mycobacteria [3]. Hence, targeting anti-ROS/RNS machinery of mycobacterial cells would facilitate efficient clearance of bacteria from the invading tissue.

The mycobacterial mel2 locus (mycobacterial enhanced infection locus, Rv1936-1941, size $7.9 \mathrm{~kb}$ ), originally identified by El-Etr et al. [6], is M. marinum and M. tuberculosis ( $\sim 98 \%$ sequence homology with M. marinum) specific and is absent in other pathogenic and nonpathogenic mycobacterial species, including M. bovis, M. avium, M. leprae and M. smegmatis, which can tolerate ROS and RNS stress responses. In silico analysis of six genes of mel2 locus, in other words melF, melG, melH, etc. revealed them to be close homologs of lux genes present in Vibrio harveyi [3]. Moreover, the melF within this locus shows high similarity to $\operatorname{lu} x A$, a monooxygenase gene involved in resistance to ROS in bioluminescent bacteria. The mel2 locus also confers resistance to ROS/RNS stress in vitro and reveals a similar function in activated murine macrophages [3,4]. Moreover, the M. marinum mel2 mutants do not display growth defect in macrophages in presence of ROS scavengers or nitric oxide synthase inhibitors, thus demonstrating that the growth defect is dependent on the production of both ROS and RNS [4]. Interestingly, the mutation in melF of mel2 locus displays a polar effect on the downstream genes of mel2 locus as the enhanced susceptibility of $m e l F$ mutant to ROS/RNS could only be partially recovered by melF alone and completely by the entire mel2 locus [3,4]. M. marinum mel2 mutants also reveal reduced bacterial growth in the late stages of mouse footpad model of infection [4]. In a similar manner, the association of mel2 locus in resistance to ROS has been demonstrated for the persistence and dissemination of $M$. tuberculosis in C57BL/6J mice [5]. The growth of $M$. tuberculosis in activated murine macrophages and infection of Phox-/- and inducible nitric oxide synthase -/- mice

Future Medicine 
as well as bone marrow derived macrophages infected with $M$. tuberculosis suggest that the primary mechanism by which mel2 impacts pathogenesis is through its ability to display resistance to ROS [5]. In addition, an enhanced production of cell wall virulence lipid, pthiocerol dimycoserosate by the M. tuberculosis mel2 mutant as compared with wild-type in presence of $\mathrm{H}_{2} \mathrm{O}_{2}$ and diamide oxidative stresses has been documented [7], thus proposing that mel2 plays a crucial role in $M$. tuberculosis lipid biosynthesis.

The development of new antitubercular drugs (ATDs) is quite difficult due to slow growth of M. tuberculosis. Alternatively, $M$. marinum, relatively a fast grower (with a generation time of $\sim 4 \mathrm{~h}$ ), requires BSL-II facility and is considered as a surrogate model to study $M$. tuberculosis pathogenesis $[6,8]$. The utility of M. marinum for evaluating the activity of ATDs has been documented [8]. Using M. marinum as an evaluation model, Liu et al. [8] identified an inhibitor targeting ICL (Rv0467) against both active and nonreplicating M. tuberculosis. Zebrafish M. marinum infection model has also been reported for evaluating ATDs in vivo as a high-throughput screening system [9]. Indeed, the TB structural genomics consortium (TBSGC), an international alliance of researchers from 15 different countries and 93 research centers, facilitates to design the 3D protein structures of $M$. tuberculosis and thus, the structure based drug designing [10]. Several protein structures are ascertained by TBSGC including malate synthase (Rv1837c), arginine biosynthetic enzymes, urease (Rv1848) and phosphoenolpyruvate carboxykinase (Rv0211), which are involved in metabolic pathways and are considered as potential drug targets. However, a crystal structure of MelF has not yet been elucidated. Therefore, we designed putative inhibitors by virtual ligand screening (VLS) targeting MelF that could diminish the ability of M. marinum to withstand ROS/RNS stress and later, the bacteriostatic/bactericidal effects of shortlisted compounds were evaluated against the M. marinum and M. tuberculosis in vitro [11]. A structure-based VLS approach was applied to the homology model of MelF protein. The ligand binding pocket was identified through consensus among multiple methods (Pocket-finder, Q-site finder and InCa-Sitefinder), and VLS was carried out by adopting a similar consensus for DOCK and Vina [11]. From the entire collection of approximately one million small drug-like molecules, a subset of approximately 250,000 inhibitors was filtered by applying Lipinski's rules of 5 for orally active drug-likeness. Furthermore, top 1000 docked inhibitors were manually screened to shortlist 178 inhibitors with good binding affinities and maximization of scaffold diversity.

M. marinum MelF, a dimeric protein $(\sim 84 \mathrm{kDa})$ exhibited flavin dependent oxidoreductase activity and the inhibitors designed by in silico analysis were screened for the reduced enzyme activity of whole bacterial cell lysate and the purified MelF [11]. Among 178 putative inhibitors evaluated, $16(\sim 9 \%)$ were found to significantly reduce the flavin oxidoreductase activity of purified MelF, while six inhibitors were shortlisted based on minimum inhibitory concentration (MIC) and minimum bactericidal concentration (MBC) values determined against the M. marinum and M. tuberculosis. Interestingly, these inhibitors did not display any nonspecific protein-structure destabilizing effects as the $\alpha$-helices and $\beta$-sheets of MelF protein were almost constant with and without inhibitors as revealed by the circular dichroism spectra evaluation [11]. It was observed that $1.68 \%(3 / 178)$ of inhibitors revealed an MBC/MIC ratio of $\leq 2$ for $M$. marinum and $M$. tuberculosis [11], which indicate better efficacy of VLS to design novel inhibitors targeting MelF, in comparison with the chemical screening with an efficacy of approximately $0.01 \%$. Moreover, few inhibitors could synergize with the first line bactericidal drugs, in other words, isoniazid (INH) and rifampicin (RIF) with no cytotoxic effect in HeLa cells [11]. Such synergistic effect could be due to generation of ROS/RNS by INH/RIF [12,13], which might have become more intense with the suppression of MelF (anti-ROS/RNS machinery). These findings suggest to exploiting new inhibitors targeting MelF with the synergistic effect, which may also improve the competence of existing ATDs. We recently demonstrated the killing of both replicating and nonreplicating M. marinum and M. tuberculosis inside the activated murine and human macrophages by such inhibitors with no cytotoxic effect [manuscript, in preparation]. Similar to MelF, $M$. tuberculosis Lsr2 (Rv3597c) appears to be a unique protein with histone-like features that could protect mycobacteria against ROS in vitro as well as during macrophage infection [14]. Also, using macrophages derived from $\mathrm{NOS}^{-/-}$ and $\mathrm{Phox}^{-1-}$ mice, Colangeli et al. [14] demonstrated that Lsr2 is crucial in protecting against ROS, but not RNS and is considered as an attractive drug target.

In a similar manner, M. tuberculosis Ddn (Rv3547) and its two homologs, in other words, Rv1261c and Rv1558 are documented to protect mycobacterial cells against ROS and bactericidal drugs such as clofazimine and INH [15]. It is proposed that the Ddn inhibitors could exhibit synergistic effect with the current ATDs, as monitored with MelF inhibitors [11]. Furthermore, Grant et al. [16] demonstrated that even a small change in dissolved oxygen concentration (20\%) could yield high quantities of hydroxyl radicals, which influence killing of mycobacterial persisters, thus confirming the susceptibility of mycobacteria to enhanced ROS. This finding seems to be critical for 
killing M. tuberculosis in hypoxic conditions, for example, granulomas. Other metabolic enzymes of M. tuberculosis, such as Lpd (Rv3303c) and DlaT are also described as drug targets, the components of pyruvate dehydrogenase and peroxynitrite redutase/peroxidase that are shown to counteract RNS induced by the host $[12,17]$. By targeting the Lpd and DlaT, Bryk et al. [12,17] identified inhibitors that are specifically effective against the nonreplicating M. tuberculosis. In addition, TMC207 (bedaquiline) and pyrazinamide have been designed by targeting the $M$. tuberculosis ATP synthase and proton motive force of the respiratory chain, respectively [11,18].

Interestingly, clofazimine and plumbagin capable of generating ROS through production of superoxide radicals are shown to reduce the MIC of INH for $M$. tuberculosis thus indicating that superoxides play a role in INH activation $[13,19]$. Since oxidative susceptibility increases susceptibility of mycobacteria to INH, there seems to be a correlation between the INH susceptibility and mel2 locus. RIF, a known RNA polymerase inhibitor, has also been demonstrated to enhance ROS including hydroxyl radicals in $M$. tuberculosis [12]. These studies provide evidence for the sensitivity of $M$. tuberculosis toward oxidative stress, however, $M$. tuberculosis's response to endogenous ROS generated within cells remains poorly understood. To understand the effects of enhanced endogenous ROS in $M$. tuberculosis, Tyagi et al. [2] synthesized a series of hydroquinone-based small molecules generating ROS. Among these, ATD-3169 permeated mycobacteria to enhance the endogenous ROS with an irreversible oxidative shift in intramycobacterial redox potential and a reduced capacity of $M$. tuberculosis to restore cytoplasmic redox balance, in comparison with $M$. smegmatis thus indicating the requisite of targeting intramycobacterial redox metabolism for controlling TB infection. Nonetheless, the inhibitors targeting MelF (anti-ROS/RNS machinery) would open up fresh avenues pertaining to the design of adequate ROS generators (both exogenous and endogenous), which may also improve the efficacy of current ATDs.

\section{Financial \& competing interests disclosure}

This work was financially supported by DBT, Govt of India, New Delhi (Project number: BT/PR5641/MED/29/557/2012) to PK Mehta and M Kulharia. R Dharra acknowledges DBT, New Delhi for providing Junior/Senior Research Fellowship and MDU, Rohtak for providing University Research Scholarship. Disclose any financial interests. The authors have no other relevant affiliations or financial involvement with any organization or entity with a financial interest in or financial conflict with the subject matter or materials discussed in the manuscript apart from those disclosed.

No writing assistance was utilized in the production of this manuscript.

\section{References}

1. Ehrt S, Schnappinger D. Mycobacterial survival strategies in the phagosome: defence against host stresses. Cell. Microbiol. 11(8), 1170-1178 (2009).

2. Tyagi P, Dharmaraja AT, Bhaskar A, Chakrapani H, Singh A. Mycobacterium tuberculosis has diminished capacity to counteract redox stress induced by elevated levels of endogenous superoxide. Free Radic. Biol. Med. 84, 344-354 (2015).

3. Subbian S, Mehta PK, Cirillo SL, Cirillo JD. The Mycobacterium marinum mel2 locus displays similarity to bacterial bioluminescence systems and plays a role in defense against reactive oxygen and nitrogen species. BMC Microbiol. 7, 4 (2007).

4. Subbian S, Mehta PK, Cirillo SLG, Bermudez LE, Cirillo JD. A Mycobacterium marinum mel2 mutant is defective for growth in macrophages that produce reactive oxygen and reactive nitrogen species. Infect. Immun. 75(1), 127-134 (2007).

5. Cirillo SLG, Subbian S, Chen B, Weisbrod TR, Jacobs WR, Cirillo JD. Protection of Mycobacterium tuberculosis from reactive oxygen species conferred by the mel2 locus impacts persistence and dissemination. Infect. Immun. 77(6), 2557-2567 (2009).

6. El-Etr SH, Subbian S, Cirillo SLG, Cirillo JD. Identification of two Mycobacterium marinum loci that affect interactions with macrophages. Infect. Immun. 72(12), 6902-6913 (2004).

7. Janagama HK, Tounkang S, Cirillo SLG, Zinniel DK, Barletta RG, Cirillo JD. Molecular analysis of the Mycobacterium tuberculosis lux-like mel2 operon. Tuberculosis 93(Suppl.), S83-S87 (2013).

8. Liu Y, Zhou S, Deng Q et al. Identification of a novel inhibitor of isocitrate lyase as a potent antitubercular agent against both active and nonreplicating Mycobacterium tuberculosis. Tuberculosis 97, 38-46 (2016).

9. Carvalho R, de Sonneville J, Stockhammer OW et al. A high-throughput screen for tuberculosis progression. PLoS ONE 6(2), e16779 (2011).

10. Chim N, Habel JE, Johnston JM et al. The TB structural genomics consortium: a decade of progress. Tuberculosis $91(2)$, 155-172 (2011).

11. Dharra R, Talwar S, Singh Y et al. Rational design of drug-like compounds targeting Mycobacterium marinum MelF protein. PLoS ONE 12(9), e0183060 (2017).

12. Bryk R, Gold B, Venugopal A et al. Selective killing of nonreplicating mycobacteria. Cell Host Microbe 3(3), 137-145 (2008). 
13. Bulatovic VM, Wengenack NL, Uhl JR, Hall L, Roberts GD, Rusnak F. Oxidative stress increases susceptibility of Mycobacterium tuberculosis to isoniazid. Antimicrob. Agents Chemother. 46(9), 2765-2771 (2002).

14. Colangeli R, Haq A, Arcus VL et al. The multifunctional histone-like protein Lsr2 protects mycobacteria against reactive oxygen intermediates. Proc. Natl Acad. Sci. USA 106(11), 4414-4418 (2009).

15. Gurumurthy M, Rao M, Mukherjee T et al. A novel F420-dependent antioxidant mechanism protects Mycobacterium tuberculosis against oxidative stress and bactericidal agents. Mol. Microbiol. 87(4), 744-755 (2013).

16. Grant SS, Kaufmann BB, Chand NS, Haseley N, Hung DT. Eradication of bacterial persisters with antibiotic-generated hydroxyl radicals. Proc. Natl Acad. Sci. USA 109(30), 12147-12152 (2012).

17. Bryk R, Arango N, Venugopal A et al. Triazaspirodimethoxybenzoyls as selective inhibitors of mycobacterial lipoamide dehydrogenase. Biochemistry 49(8), 1616-1627 (2010).

18. Mdluli K, Kaneko T, Upton A. The tuberculosis drug dscovery and development pipeline and emerging drug targets. Cold Spring Harb. Perspect. Med. 5(6), a021154 (2015).

19. Yano T, Kassovska-Bratinova S, Shin Teh J et al. Reduction of clofazimine by mycobacterial type 2 NADH: quinone qxidoreductase: a pathway for the generation of bactericidal levels of reactive oxygen species. J. Biol. Chem. 286(12), 10276-10287 (2011). 